\title{
DESIGN OF DOUBLY-COMPLEMENTARY IIR DIGITAL FILTERS, USING A SINGLE COMPLEX ALLPASS FLTER
}

\author{
P. P. VAIDYANATHAN, ${ }^{1}$ P. REGALIA, ${ }^{2}$ AND S. K. MTRA ${ }^{2}$ \\ ${ }^{1}$ Dept. of EE, Caltech, Pasadena, CA 91125, USA \\ ${ }^{2}$ Dept. of ECE, Univ. of Calif., Santa Barbara, CA 93106, USA
}

\begin{abstract}
It is shown that a wide class of real-coefficient, doubly-complementary IIR trassfer-function pairs can be implemented by means of a single complex allpass filter. For a real input sequence, the real part of the output sequence of the complex allpass filter corresponds to one of the transfer functions $G(z)$ (for example, lowpass), whereas the imaginary part of the output sequence corresponds to its "complementary" filter $H(z)$ (for example, highpass). Since the resulting implementation is structurally lossless, $G(z)$ and $H(z)$ have very low passband-sensitivity. Numerical design examples are included to demonstrate the ideas.
\end{abstract}

\section{INTRODUCTION}

In this psper we consider digital infinite impulse response (IIR) stable transfer functions of the form

$$
G(z)=P(z) / D(z)=\sum_{n=0}^{N} p_{n} z^{-n} / \sum_{n=0}^{N} d_{n} z^{-n}
$$

where $p_{n}$ and $d_{n}$ are real. Without loss of generality, it is assumed that $\left|G\left(e^{j \omega}\right)\right| \leq 1$ for all $\omega$, i.e., we assume $G(z)$ to be a BR function [1]. A transfer function which is "complementary" to $G(z)$ is any function $H(z)=$ $Q(z) / D(z)$ such that

$$
\left|H\left(e^{j \omega}\right)\right|^{2}+\left|G\left(e^{j \omega}\right)\right|^{2}=1
$$

for all $\omega$. Equation (2) is called the power complementary property. In a number of filtering applications, the numerators $P(z)$ and $Q(z)$ have linear-phase (a common reason being that the transmission zeros of $G(z)$ and $H(z)$ are typically on the unit circle). Accordingly, these polynomials are symmetric or antisymmetric. When $P(z)$ is symmetric and $Q(z)$ is antisymmetric, it is well known [2],[3] that $G(z)$ and $H(z)$ can be implemented in the form

$$
\begin{aligned}
& G(z)=\frac{1}{2}\left[A_{1}(z)+A_{2}(z)\right] \\
& H(z)=\frac{1}{2}\left[A_{1}(z)-A_{2}(z)\right]
\end{aligned}
$$

where $A_{1}(z)$ and $A_{2}(z)$ are stable allpass functions with real coefficients. If $N_{1}$ and $N_{2}$ denote the orders of $A_{1}(z)$ and $A_{2}(z)$ then $N_{1}+N_{2}=N$. Implementation of $G(z)$ and $H(z)$ in terms of allpass functions in the above manner leads to extremely low passband-sensitivities because of structural boundedness [1],[2]. Moreover, a total of $\boldsymbol{N}$ multipliers is sufficient in order to generate both the transfer functions $G(z)$ and $H(z)$, and accordingly we have a very efficient implementation.

A wide class of transfer functions fall under the class which can be implemented as above. Typical examples are odd order Butterworth, Chebyshev, and elliptic digital low pass filters. Now, equations (3),(4) are equivalent to the inverse equations

$$
\begin{aligned}
& A_{1}(z)=G(z)+H(z) \\
& A_{2}(z)=G(z)-H(z) .
\end{aligned}
$$

Any pair of transfer functions satisfying (2) and (5) is said to be a "doubly-complementary" pair.

In the above discussion, $P(z)$ was assumed symmetric and $Q(z)$ antisymmetric. Next, if both $P(z)$ and $Q(z)$ are symmetric, ${ }^{\ddagger}$ the above allpass decomposition is not valid. This situation arises when neither $G(z)$ nor $H(z)$ has a zero at $\omega=0$. Typical examples are even order digital Chebyshev and elliptic lowpass and highpass filters. In the next section we show how a different type of decomposition can be performed in terms of complex allpass functions.

\section{THE DECOMPOSITION OF A BR FUNCTION INTO COMPLEX ALLPASS SECTIONS}

Let $G(z)$ be a BR function as in (1). Assume there are no uncancelled common factors between $P(z)$ and $D(z)$. Let $H(z)$ be the complementary BR function satisfying (2) and given by

$$
H(z)=Q(z) / D(z)=\sum_{n=0}^{N} q_{n} z^{-n} / \sum_{n=0}^{N} d_{n} z^{-n} .
$$

$\ddagger$ It is not possible for both $P(z)$ and $Q(z)$ to be antisymmetric, for this would imply that the left side of (2) is zero at $\omega=0$.

48. 4. 1 


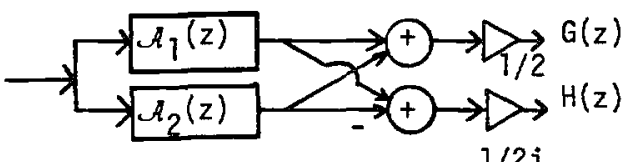

$1 / 2 j$

Fig. 1. Implementing the complementary pair. $\underset{(\text { real })}{x(n)} \rightarrow f_{i}(z) \stackrel{y(n)}{\longrightarrow} \longrightarrow{ }^{y_{r}(n)} G(z)=\frac{Y_{r}(z)}{X(z)}$

Fig. 2. Realizing $G(z)$ by one allpass function.

The coefficients $p_{n}, q_{n}$, and $d_{n}$ are all real-valued. Assuming the numerators $P(z)$ and $Q(z)$ to be symmetric polynomials, we have $p_{n}=p_{N-n}$ and $q_{n}=q_{N-n}$, and hence we have $\ddagger$

$$
\tilde{P}(z)=z^{N} P(z), \tilde{Q}(z)=z^{N} Q(z)
$$

If the power-complementary property of (2) holds, then by analytic continuation we have

$$
\tilde{P}(z) P(z)+\tilde{Q}(z) Q(z)=\widetilde{D}(z) D(z)
$$

for all $z$. In view of (8), this becomes

$$
P^{2}(z)+Q^{2}(z)=z^{-N} D\left(z^{-1}\right) D(z)
$$

which can be rewritten as

$$
[P(z)+j Q(z)][P(z)-j Q(z)]=z^{-N} D\left(z^{-1}\right) D(z)
$$

With three polynomials $P(z), Q(z)$, and $D(z)$ with realcoefficients satisfying (11), it can be shown that $D(z)$ cannot have real zeros when there are no common factors between $P(z)$ and $D(z)$ (as assumed). Accordingly all zeros of $D(z)$ are complex conjugate pairs, and hence $\boldsymbol{N}$ must be even and $D(z)$ has the form

$$
D(z)=\prod_{k=1}^{M}\left(1-z^{-1} z_{k}\right)\left(1-z^{-1} z_{k}^{*}\right), M=\frac{N}{2} .
$$

Thus, (11) becomes

$[P(z)+j Q(z)][P(z)-j Q(z)]=$

$z^{-N} \prod_{k=1}^{M}\left(1-z^{-1} z_{k}\right)\left(1-z^{-1} z_{k}^{*}\right)\left(1-z z_{k}\right)\left(1-z z_{k}^{*}\right)$.

Since $\stackrel{k}{P}(z)$ and $Q(z)$ are symmetric, the zeros of $P(z)+$ $j Q(z)$ occur in reciprocal pairs. The same is true of $P(z)-j Q(z)$. Moreover, if $z_{k}$ is a zero of $P(z)+j Q(z)$, then $z_{k}^{*}$ is a zero of $P(z)-j Q(z)$ because the coefficients in $P(z)$ and $Q(z)$ are real. Consequently, we can uniquely factorize (13) as

$\ddagger$ The tilde notation means that $z$ should be replaced with $z^{-1}$, and the coefficients (if complex) should be conjugated. On the unit circle, this corresponds to conjugating the function itself.

$$
\begin{aligned}
& P(z)+j Q(z)=\beta z^{-M} \prod_{k=1}^{M}\left(1-z^{-1} z_{k}\right)\left(1-z z_{k}\right) \\
& P(z)-j Q(z)=\beta^{*} z^{-M} \prod_{k=1}^{M}\left(1-z^{-1} z_{k}^{*}\right)\left(1-z z_{k}^{*}\right)
\end{aligned}
$$

where $\beta$ is a complex constant (to be identified below). Dividing both sides of $(14),(15)$ by $D(z)$, we thus arrive at

$$
\begin{aligned}
& G(z)+j H(z)=A_{1}(z) \\
& G(z)-j H(z)=A_{2}(z)
\end{aligned}
$$

where $A_{1}(z)$ and $A_{2}(z)$ are stable allpass functions with complex coefiicients, defined as

$$
A_{1}(z)=\beta \prod_{k=1}^{M} \frac{z^{-1}-z_{k}}{1-z^{-1} z_{k}^{*}}, A_{2}(z)=\beta^{*} \prod_{k=1}^{M} \frac{z^{-1}-z_{k}^{*}}{1-z^{-1} z_{k}} .
$$

The inverses of the relations (16),(17) are

$$
\begin{aligned}
& G(z)=\left[A_{1}(z)+\mathscr{A}_{2}(z)\right] / 2 \\
& H(z)=\left[A_{1}(z)-A_{2}(z)\right] / 2 j .
\end{aligned}
$$

In summary, $G(z)$ and $j H(z)$ satisfy (2) and (16) simultaneously, and hence form a doubly-complementary pair. Moreover, they can be implemented in terms of sums and differences of allpass functions. Figure 1 shows a schematic of the implementation.

The allpass functions $A_{1}(z)$ and $A_{2}(z)$ have orders $M$ each, where $M=N / 2$. The coefficients of these functions are complex, and moreover the coefficients of $\mathcal{A}_{2}(z)$ are the complex conjugates of those of $A_{1}(z)$. Accordingly, with real inputs, in order to realize the schematic of Figure 1 , it is only necessary to implement $h_{1}(z)$. The real part of the output sequence corresponds to the output of $G(z)$ and the imaginary part corresponds to the output of $H(z)$. Figure 2 shows this implementation. The simplest means of implementing $A_{1}(z)$ is to cascade first-order sections of the form shown in Figure 3. Four real multiplications are involved in the circuit of Figure 3. The multiplier $\beta$ in (18), operating on

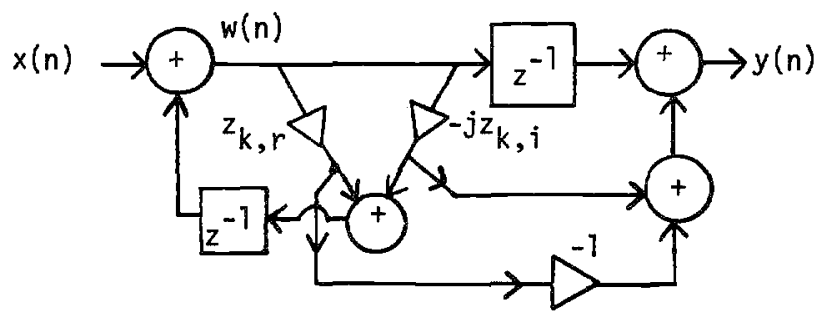

Fig. 3. Implementing a complex allpass section. 
a real input sequence, is equivalent to two real multiplications. Thus the total number of real multiplications per transfer function in Figure 2 is $(4 M+2) / 2=N+1$, as against the direct form which requires $3 N / 2+1$ real multiplications for a linear-phase numerator.

Since the multipliers in Figure 2 can be quantized such that $A_{1}(z)$ remains stable allpass, it is clear that $G(z)$ and $H(z)$ remain BR in spite of quantization. Accordingly, the implementation exhibits low passbandsensitivity $[1],[2]$, and remains stable in spite of multiplier quantization.

It is also possible to obtain cascaded lattice implementations of (18) by employing a complex version of the Gray and Markel lattice structures [4]. Furthermore, a large number of equivalent structures for complex allpass functions can be generated by appropriate extension of the multiplier extraction approach [5]. The relative merits of these various schemes remain to be explored:

\section{A DESIGN EXAMPLE}

Given a BR function $G(z)=P(z) / D(z)$, with symmetric $P(z)$, assume that there exists $H(z)=$ $Q(z) / D(z)$ with symmetric numerator $Q(z)$, satisfying (2). Then $Q(z)$ can be computed easily without going through elaborate spectral factorization schemes, by noting that (10) implies

$$
Q^{2}(z)=z^{-N} D\left(z^{-1}\right) D(z)-P^{2}(z) .
$$

Let the righthand side of (21), which is known, be denoted as $R(z)=\sum_{k=0}^{2 N} r_{k} z^{-k}$. Then the coefficients of $Q(z)$ can be recursively computed as

$$
\begin{aligned}
& q_{0}=\sqrt{r_{0}}, q_{1}=r_{1} / 2 q_{0} \\
& q_{n}=\left(r_{n}-\sum_{k=1}^{n-1} q_{k} q_{n-k}\right) / 2 q_{0}, \quad 2 \leq n \leq \frac{N}{2} \\
& q_{n}=q_{N-n}, n>\frac{N}{2} .
\end{aligned}
$$

Once $Q(z)$ is found, we evaluate the zeros of the polynomial $P(z)+j Q(z)$, in order to identify $z_{k}$ in (14). In order to find $\beta$, note that from (16)

$$
G(1)+j H(1)=\beta \prod_{k=1}^{M} \frac{1-z_{k}}{1-z_{k}^{*}} .
$$

Since all quantities in (25) except $\beta$ are known, $\beta$ can be evaluated. Thus, $\boldsymbol{A}_{1}(z)$ has been completely identified, and the implementation of Figure 2 can be obtained.

As an example, consider the design of an 8th-order lowpass elliptic BR digital transfer function:

$$
\begin{aligned}
& G(z)=\frac{P(z)}{D(z)}= \\
& \frac{0.026-0.029 z^{-1}+0.074 z^{-2}-0.042 z^{-3}+0.084 z^{-4}}{1-3.982 z^{-1}+8.498 z^{-2}-11.486 z^{-3}+10.707 z^{-4}} \\
& \frac{-0.042 z^{-5}+0.074 z^{-6}-0.029 z^{-7}+0.026 z^{-8}}{-6.906 z^{-5}+3.019 z^{-6}-0.809 z^{-7}+0.104 z^{-8}}
\end{aligned}
$$

Once $Q(z)$ is computed, we have

$$
\begin{aligned}
& H(z)=\frac{Q(z)}{D(z)}= \\
& \frac{0.321-1.901 z^{-1}+5.455 z^{-2}-9.724 z^{-3}+11.706 z^{-4}}{1-3.982 z^{-1}+8.498 z^{-2}-11.486 z^{-3}+10.707 z^{-4}} \\
& \frac{-9.724 z^{-5}+5.455 z^{-6}-1.901 z^{-7}+0.321 z^{-8}}{-6.906 z^{-5}+3.019 z^{-6}-0.809 z^{-7}+0.104 z^{-8}}
\end{aligned}
$$

We then compute the zeros of $P(z)+j Q(z)$ and identify $\boldsymbol{A}_{1}(z)$ :

$$
A_{1}(z)=\beta \prod_{k=1}^{4} \frac{z^{-1}-z_{k}}{1-z^{-1} z_{k}^{*}}
$$

where

$$
\beta=0.4698+j 0.8828
$$

$$
\begin{aligned}
& z_{1}=0.4344-j 0.2253, z_{2}=0.4831+j 0.5675 \\
& z_{3}=0.5244-j 0.7367, z_{4}=0.5492+j 0.8075 .(30)
\end{aligned}
$$

In order to demonstrate the low passband-sensitivity properties, the structure of Figure 2 was simulated digitally, with quantized multipliers. The allpass function $A_{1}(z)$ was implemented by cascading the first-order sections in Figure 3; each multiplier $z_{k, r}$ and $z_{k, i}$ was quantired to 4 binary bits in sign-digit code.

Figure 4 shows the magnitude responses of $H(z)$ and $G(z)$ in the ideal (i.e., unquantized) case. Figure 5 shows the response $\left|G\left(e^{j \omega}\right)\right|$ for the 4-bit allpassbased implementation of Figure 2. The low passbandsensitivity is evident from the plot of passband details, which are included in Figure 5(b). Next, the same transfer function $G(z)$ was implemented in direct-form with 9-bits per multiplier. The corresponding responses are shown in Figure 6 which reveals a very poor passbandsensitivity. Thus, a 4-bit allpass based implementation (Figure 2) has much better passband response than a 9-bit direct-form implementation. In addition, the implementation of Figure 2 requires only 9 real multipliers per transfer function, whereas the direct-form requires 13 multipliers. 


\section{CONCLUDING REMARKS}

A new class of structurally passive low-sensitivity implementations has been reported in this paper. The implementation is a single complex allpass section, giving rise to two BR transfer functions. Application of these results in signal-splitting and reconstruction, and in multi-rate signal processing are currently being studied.

\section{References}

[1] P. P. Vaidyanathan and S. K. Mitra, "Low passband sensitivity digital filters: A generalized viewpoint and synthesis procedures," Proceedings of the IEEE, pp. 404-423, Apr. 1984.

[2] P. P. Vaidyanathan, S. K. Mitra, and Y. Neuvo, "A new approach to the realization of low sensitivity IIR digital filters," IEEE Trans. on Acoustics, Speech, and Signal Processing, v. ASSP-34, 1986 (to appear).

[3] A. Fettweis, "Wave digital lattice filters," Int. J. Circuit Theory and Applications, pp. 203-211, June 1974.

[4] A. H. Gray and J. D. Markel, "Digital lattice and ladder filter synthesis," IEEE Trans. Audio Electroacoustics, v. AU-21, pp. 491-500, Dec. 1973.

[5] S. K. Mitra and K. Hirano, "Digital allpass networks," IEEE Trans. on Circuits and Systems, v. CAS21, pp. 688-700, Sept. 1974.

[6] A. G. Constantinides and R. A. Valenzuela, "An efficient and modular transmultiplexer design," IEEE Trans. on Comm., v. 30, pp. 1629-1641, July 1982.

(7) R. Ansari and B. Liu, "A class of low-noise computationally efficient recursive digital filters with applications to sampling rate alterations," IEEE Trans. Acoustics, Speech, and Signal Proc., pp. 90-97, Feb. 1985.

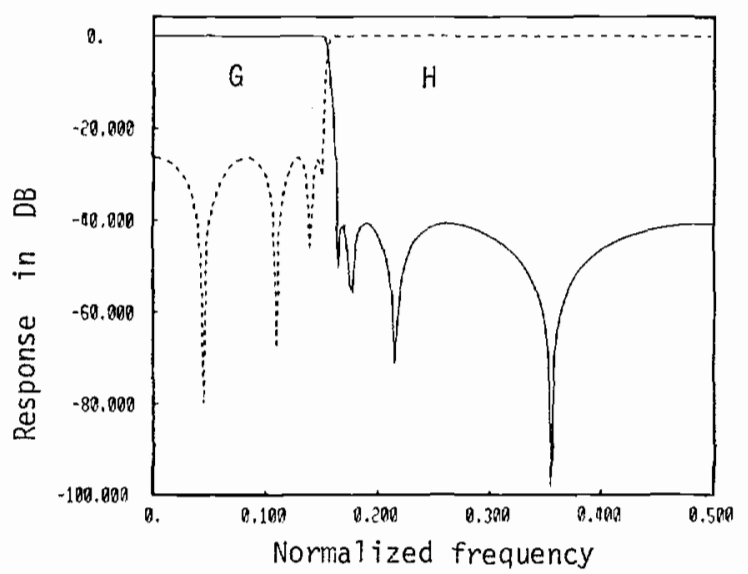

Fig. 4. The conplementary responses.

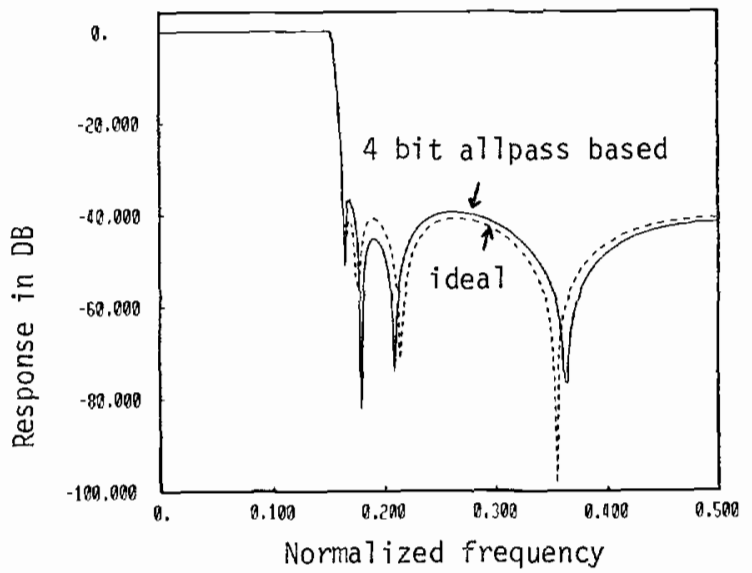

Fig. 5(a) The quantized implementation.

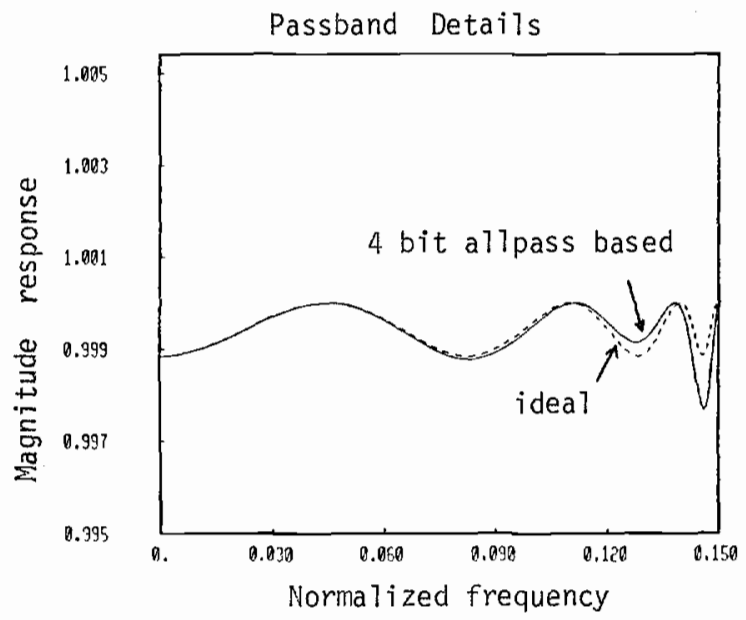

Fig. 5(b) The quantized implementation.

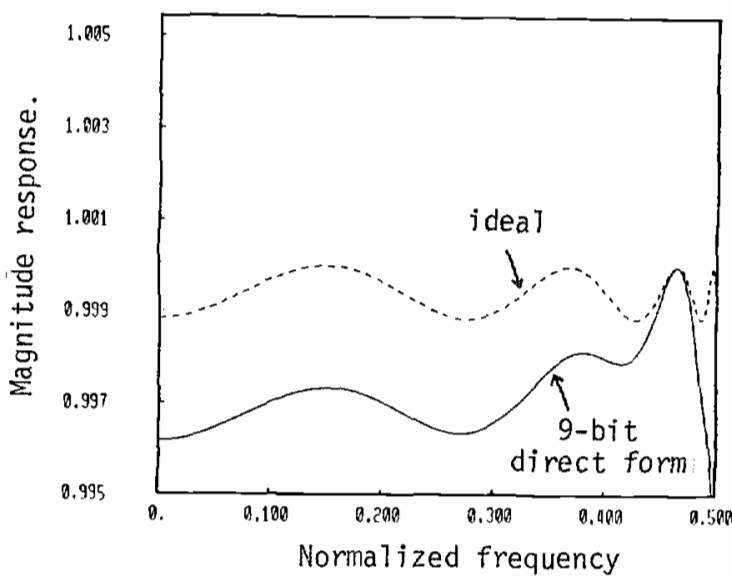

Fig. 6. The quantized direct form. Work supported in part by NSF grants ECS $84-04245$ and ECS $85-08017$ and in part by Caltech's PAT grant.

48. 4. 4 